\title{
Reusable Chemically-Micropatterned Substrates via Sequential Photoinitiated Thiol-Ene Reactions as Template for Perovskite Thin-Film Microarrays
}

\author{
Kurt Waldo E. Sy Piecco ${ }^{\mathrm{a}, \mathrm{c}, \mathrm{d}}$, Juvinch R. Vicente ${ }^{\mathrm{a}, \mathrm{c}, \mathrm{d}}$, Joseph R. Pyle ${ }^{\mathrm{a}, \mathrm{c}}$, David C. Ingram ${ }^{\mathrm{b}, \mathrm{c}}$, \\ Martin E. Kordesch ${ }^{\mathrm{b}, \mathrm{c}}$, Jixin Chen ${ }^{\mathrm{a}, \mathrm{c} *}$ \\ ${ }^{\mathrm{a}}$ Department of Chemistry and Biochemistry, ${ }^{\mathrm{b}}$ Department of Physics and Astronomy, \\ ${ }^{c}$ Nanoscale and Quantum Phenomena Institute, Ohio University, Athens, OH 45701, USA \\ ${ }^{\mathrm{d}}$ University of the Philippines Visayas, Miagao, Iloilo 5023, Philippines
}

*Corresponding author: chenj@ohio.edu 


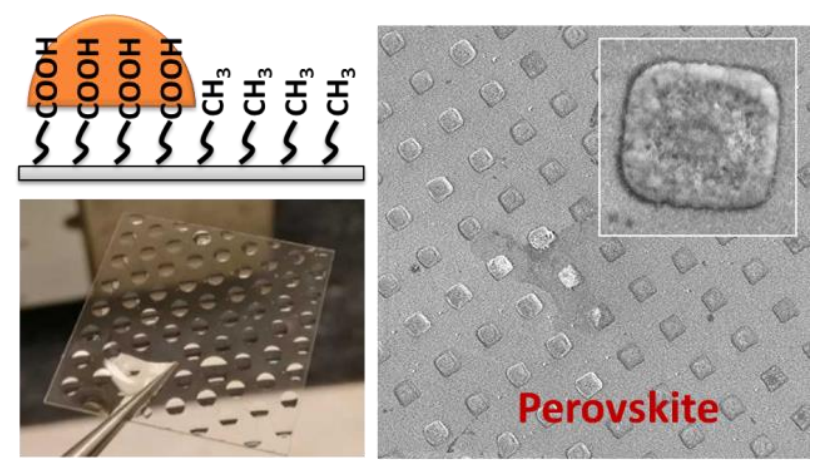

\begin{abstract}
Patterning semiconducting materials are important for many applications such as microelectronics, displays, and photodetectors. Lead halide perovskites are an emerging class of semiconducting materials that can be patterned via solution-based methods. Here we report an allbenchtop patterning strategy by first generating a patterned surface with contrasting wettabilities to organic solvents that have been used in the perovskite precursor solution then spin-coating the solution onto the patterned surface. The precursor solution only stays in the area with higher affinity (wettability). We applied sequential sunlight-initiated thiol-ene reactions to functionalize (and pattern) both glass and conductive fluorine-doped tin oxide (FTO) transparent glass surfaces. The functionalized surfaces were measured with the solvent contact angles of water and different organic solvents and were further characterized by XPS, selective fluorescence staining, and selective DNA adsorption. By simply spin-coating and baking the perovskite precursor solution on the patterned substrates, we obtained perovskite thin-film microarrays. The spin-coated perovskite arrays were characterized by XRD, AFM, and SEM. We concluded that Patterned substrate prepared via sequential sunlight-initiated thiol-ene click reactions is suitable to fabricate perovskite arrays via the benchtop process. In addition, the same patterned substrates can be reused
\end{abstract}


several times until a favorable perovskite microarray is acquired. Among a few conditions we have tested, DMSO solvent and modified FTO surfaces with alternatively carboxylic acid and alkane is the best combination to obtain high-quality perovskite microarrays. The solvent contact angle of DMSO on carboxylic acid-modified FTO surface is nearly zero and $65 \pm 3^{\circ}$ on octadecane modified FTO surface.

Keywords: thiol-ene click chemistry, visible-light photopatterning, FTO glass surface functionalization, solvent contact angles, peovksite microarrays

\section{Introduction}

Organic-inorganic hybrid perovskites (OHPs) are materials with a chemical formula of $\mathrm{ABX}_{3}$. For example, in $\mathrm{MAPbX}$, methylammonium (MA) ions, lead ions, and halides $\left(\mathrm{Cl}^{-}, \mathrm{Br}^{-}\right.$or $\mathrm{I}^{-}$) occupy the A, B, and X sites in the OHP crystal lattice, respectively. The surge of interests in these materials is largely due to their excellent optoelectronic properties and their low-cost solution-based processing that lead to the development of new solar cells, light emitting devices, lasers, sensors, and etc. ${ }^{1-7}$ Micropatterned perovskite thin films are necessary for miniaturized integrated optoelectronic systems. In the literature, both bottom-up and top-down methods have been employed to fabricate a patterned array of perovskites. Bottom-up approaches have been explored, such as printing perovskite patterns through inkjet and roller printing, ${ }^{8-10}$ or using patterned PDMS molds and packed colloidal spheres for growing perovskite crystals in confined spaces. ${ }^{11-18}$ Various other templates have also been used, such as anodized aluminum oxide membranes and patterned $\mathrm{TiO}_{2}$ films for the confined crystallization of perovskite arrays. ${ }^{19-21}$ Topdown approaches, such as photolithography, e-beam lithography, and atomic force microscopy 
have been employed. ${ }^{22,23}$ Conventional photolithography involves photoresist that is patterned and developed on top of the perovskite layer followed by etching of exposed perovskite thin films. ${ }^{24,25}$ This method has been modified by pre-patterning the substrates followed by the spin coating of perovskite precursors to avoid the step to lift-off the photoresist. ${ }^{26-31}$ Although these top-down methods provide good quality patterned thin films, several steps involved in the patterning and curing of the photoresist, lift-off, and etching drive up the cost and processing time of fabrication and affect the perovskite properties that jeopdize scaling-up. High-quality, low-cost, and yet simple micropatterning techniques are still highly desired.

We rationalize that simple benchtop surface patterning method, such as thiol-ene click chemistry, ${ }^{32,33}$ may provide a chemically-patterned substrate for the spin-coating of perovskite precursors. The excellent quality of thin films from the spin-coating process has been demonstrated in the literature. ${ }^{34}$ In addition, literature reports have shown that by properly introducing organic self-assembled layers at the interfaces, the performance of perovskite solar cells can be greatly enhanced. ${ }^{34-36}$ The thiol-ene click reaction has been studied extensively in the literature to be an orthogonal coupling reaction between any alkenes and thiols. ${ }^{37}$ The photo-initiated thiol-ene click reaction produces high yields even in the presence of water and oxygen, which greatly simplifies the process. ${ }^{38}$ In our previous work, ${ }^{32}$ we have studied the photochemical kinetics of the reaction between vinyltrimethoxysilane (VTMS) with either thioglycolic acid (TGA) or cysteamine hydrochloride $(\mathrm{CAH})$ under sunlight. This reaction can be directly adapted in the surface functionalization of oxides surfaces such as glass and fluorine-doped tin oxide (FTO) substrates.

In this report, we have fabricated perovskite thin film arrays on reusable thiol-enechemically-patterned glass and FTO substrates. The oxide surface is initially functionalized using VTMS uniformly then patterned by sequential sunlight-initiated thiol-ene reactions under a 
photomask. The first thiol-ene reaction results in the grafting of TGA (or CAH) molecules to the surface, thus producing hydrophilic regions on the substrate. In the second thiol-ene treatment step, octadecane thiol (ODT) is used to produce hydrophobic regions. These surfaces show distinguishing affinities (wettability) to water and many organic solvents. The wetting hysteresis behavior of the perovskite precursor solution on the patterned hydrophilic/hydrophobic surface provides a simple method to fabricate perovskite thin film arrays under ambient conditions with a single spin-coating step. Furthermore, the chemically-patterned substrate can be reused several times after sonicating off the old film.

\section{Results and Discussion}

Detailed sample preparation procedures have been described in the experimental section of the supporting information (SI). Briefly, the cleaned glass or fluorine-doped tin oxide (FTO) glass substrate was first functionalized with vinyltrimethoxysilane (VTMS). Then different thiols, namely cysteamine hydrochloride (CAH), thioglycolic acid (TGA), and octadecanethiol (ODT) are patterned onto the substrate via photoinitiated thiol-ene click reaction using photomasks and simulated sunlight (Figure 1). The idea is to test the effect of solvent affinity and surface functional group (such as surface charge and acidity) on the feasibility of surface patterning and the quality of the perovskite arrays. We have reported the reaction kinetics of these reactions in the bulk solution under the same conditions very recently. ${ }^{32}$ We characterized these patterned samples using XPS and fluorescence microscope by selectively labeling the surfaces. Then we used the patterned substrate to fabricated perovskite microarrays using the simple spin-coating method. Both functionalized glass and FTO glass substrates show consistent properties in perovskite patterning. 
We will focus our discussion more on FTO substrates because they are more interesting for optoelectronic applications than glass.

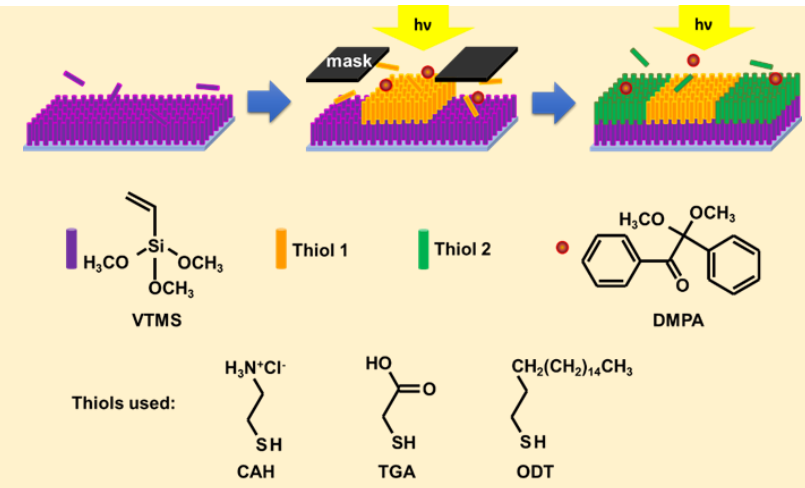

Figure 1. Scheme of the patterning procedure for the stepwise thiol-ene coupling reactions on the surface of a vinyl-functionalized substrate with 2,2-dimethoxy-2-phenylacetophenone (DMPA) added as the photoinitiator. CAH or TGA was used in the first thiol treatment, while ODT is used during the second thiol treatment step.

\section{Physico-Chemical Properties of Chemically-Patterned Substrates}

Water contact angles are simple and distinct indications of the successful surface modifications. Figure 2 clearly shows the effectiveness of each thiol-ene coupling reaction step. When the oxygen plasma-treated FTO was coated with vinyl groups, the static water contact angle changed from near $0^{\circ}$ (super hydrophilic, noted as wet) to $109 \pm 1^{\circ}$ because the vinyl group is nonpolar and hydrophobic. The latter value is significantly higher than the literature value $76^{\circ}$ for a vinyl-modified surface ${ }^{39}$ due to the roughness of the FTO substrate, which can trap air bubbles in the crevices. ${ }^{40}$ When the vinyl-substrate was modified with TGA or CAH, water angles decrease. The carboxylic acid moiety in TGA $\left(\mathrm{pKa}_{1}(-\mathrm{COOH})=4.32, \mathrm{pKa}_{2}(-\mathrm{SH})=10.2\right)^{41,42}$ can hydrogen bond with water molecules, and are partially deprotonated. The ammonium chloride moiety in CAH $\left(\mathrm{pKa}_{1}(-\mathrm{SH})=8.27, \mathrm{pKa}_{2}\left(-\mathrm{NH}_{2}\right)=10.53\right)^{41,43}$ ionizes and interacts favorably with water. In both cases, the static contact angle will decrease after the vinyl-substrate is treated with either TGA or CAH. From a static water contact angle of $109^{\circ}$ on a vinyl-functionalized surface, the contact 
angle was reduced significantly to $46 \pm 3^{\circ}$ for a carboxyl-functionalized surface, and to $68 \pm 4^{\circ}$ for an amine-functionalized surface. The contact angle of the FTO-ODT substrate was compared against an equivalently-functionalized surface using a solution of trichloro-octadecylsilane (ODS) in toluene and showed very similar values, which indicates the possible similarities in their monolayer brush arrangement on the surface. ${ }^{44,45}$

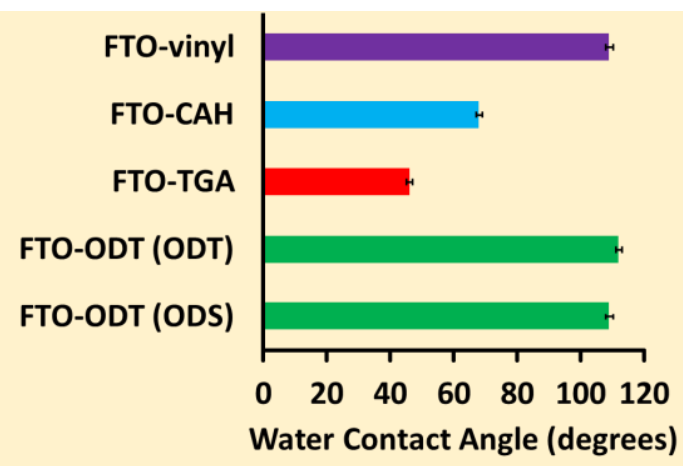

Figure 2. Static water contact angles of the functionalized FTO-substrates. Error bars are the standard deviation of 9 measurements of 3 independent samples. See supporting information for the data and example images.

Direct conformation of the click chemistry on the glass surface is then obtained from the $\mathrm{x}$-ray photoelectron spectra (XPS) (Figure 3) using both $\mathrm{Al}$ and $\mathrm{Mg}$ sources (Figure 3B). The peak assignments are based on the XPS database from the National Institute of Standards and Technology. ${ }^{46}$ XPS peaks form the glass components Na (1072, 64, 30, eV; Auger $\left.988 \mathrm{eV}\right), \mathrm{Si}$ $(1844,150,102 \mathrm{eV}), \mathrm{O}(532,25, \mathrm{eV})$ are significant on the survey spectra (Figure 3A, 3B). Small carbon and sulfur contamination are observed in all samples. XPS peaks from the organic functional layer $\mathrm{N}(400 \mathrm{eV})$ and $\mathrm{C}(284.8$, and $288 \mathrm{eV})$ can be observed in the accumulated fine scans (Figure 3C, 3D). The oxidized carbon contamination on VTMS treated glass ( $288 \mathrm{eV}$ peak in Figure 3C) is likely to be negatively charged which is removed by the positively charged CAH and appears again in TGA modified surface. This is because TGA has a $\mathrm{COOH}$ group that has a C1s peak of around $288 \mathrm{eV}$. The nitrogen peak observed on the surface of VTMS-CAH modified 
glass absent on the VTMS-TGA glass is a strong indicator of the successful thiol-ene coupling reaction (Figure 3D).

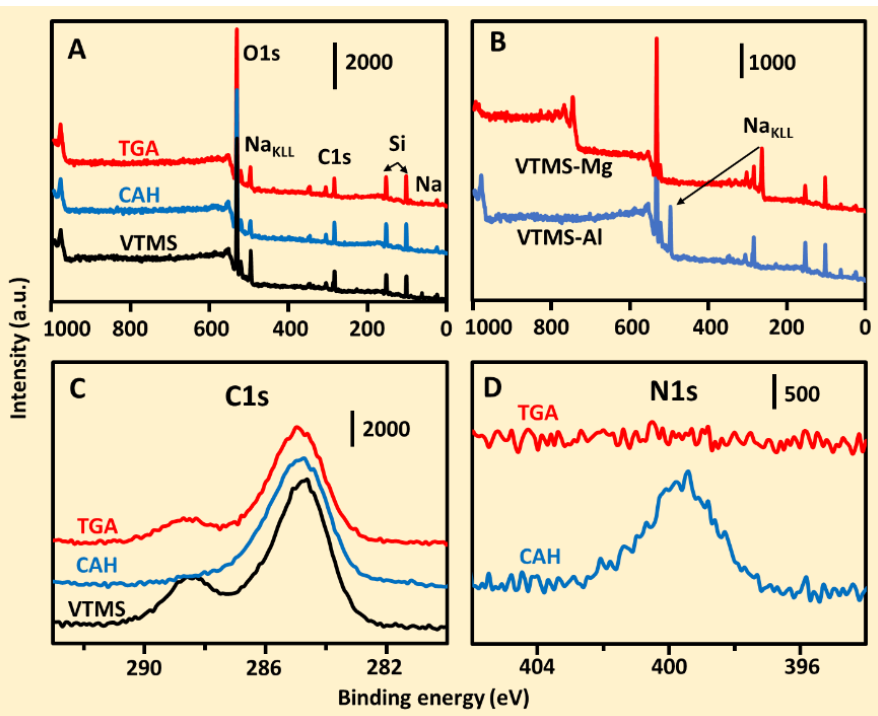

Figure 3. (A) XPS survey spectra of the Glass-vinyl, Glass-CAH, and Glass-TGA modified glass substrates. (B) Survey spectra of Glass-vinyl modified glass using Al and Mg X-ray source. The (C) C1s, and (D) N1s spectra of the substrates obtained using Al source.

The production of a chemically patterned substrate was validated by labeling the surfacebound amine moieties with a fluorescent ATTO 633 NHS-ester dye via EDC-NHS click chemistry, one of the mostly used covalent labeling methods. ${ }^{47,48}$ For this portion, a vinyl-functionalized glass substrate was used to see if the surface bound thiols from a previous thiol-ene treatment step will affect the reaction of the second thiol to the remaining surface vinyl-groups. More specifically, we want to check if the presence of amine or carboxylic acid groups on the substrate will affect the surface-localized photoinitiated thiol-ene reaction.

Figures 4A and 4B show the good resolution achievable by our method when comparing the fluorescently labelled pattern with the TEM copper grid photomask (Figure SI-4A) that has square-shaped holes. Therefore, if the vinyl-functionalized substrate is initially treated with TGA 
followed by $\mathrm{CAH}$, the fluorescent pattern generated will be the one shown in Figure 4A. Since the ATTO 633 dye has aromatic moieties, it is possible for the dye to be simply adsorbed on unreacted vinyl-functionalized regions (Figure SI-4C) and still generate the image in Figure 4A. To test if consecutive thiol-ene reactions are possible on the same substrate, we reversed the sequence of the thiol treatment. The negative of the pattern is shown in Figure 4B, which is an image of a vinyl-functionalized substrate treated first with CAH then with TGA. If the second treatment with TGA did not work, then the whole surface would be fluorescent because it would have both vinyl and amine areas. If the first treatment with $\mathrm{CAH}$ did not work, then the whole surface would have been functionalized with carboxylic acids during the second treatment step (because there is no photomask used in the second step), and the whole substrate would not be fluorescent. Since both treatment sequences generated the expected results, consecutive thiol-ene reactions on the same substrate have worked. Thus, the microscope images of the dye labeled patterned substrates in Figures 4A and 4B shows proof of the orthogonality of click reactions on the substrate surface. However, we should note that the first step reaction has not reached surface saturation under our experimental conditions thus there is a small amount of unreacted vinyl left on the surface which causes the background signal in Figures 4A and 4B. We did not use FTOCAH-ODT substrates in this experiment since the ATTO633 dye will also likely adsorb on the ODT-functionalized surfaces, similar to the vinyl-functionalized surfaces, which will not give us conclusive results. In summary, we have clearly shown that, in this case, the presence of the surface-bound thiol in the first thiol-ene treatment does not affect the reaction of the second thiol with the remaining vinyl groups on the substrate. 


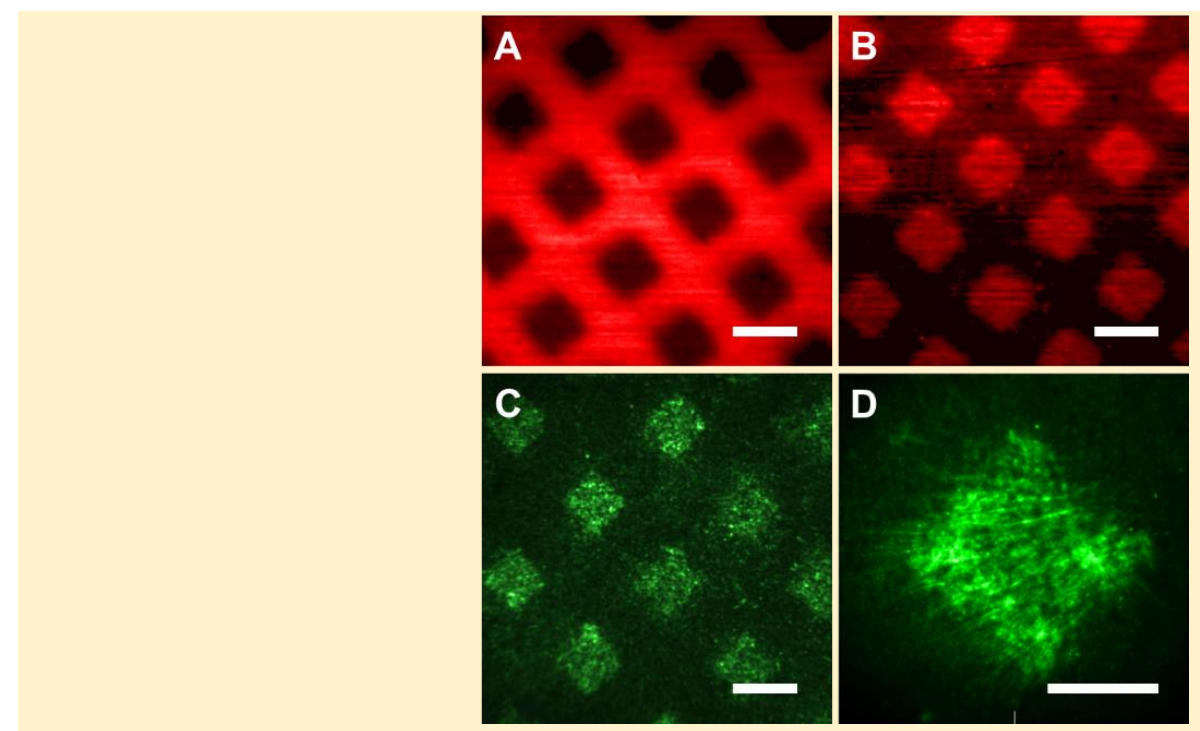

Figure 4. (A and B) Fluorescence microscope images of patterned glass substrates labeled with ATTO-633-NHS dye (red). (A) The vinyl-functionalized glass was treated first with TGA, then CAH. (B) The vinyl-functionalized glass was treated first with CAH followed by TGA. Combed $\lambda$-DNA (green) on a Glass-CAH-TGA substrate under (C) $20 \times$ and (D) $100 \times$ magnification. The combing direction was from right to left. Scale bars $=40 \mu \mathrm{m}$ in $\mathrm{A}, \mathrm{B}$, and C; $20 \mu \mathrm{m}$ in D.

Due to the negatively-charged phosphate groups along the backbones of DNA molecules, strands of DNA can be immobilized and stretched on positively-charged surfaces. Thus, we further demonstrate the feasibility of making patterned positive-negative charged surfaces, as shown in Figures 4C and 4D. For this experiment, we have utilized a patterned glass substrate that was the same as the patterned substrate used in Figure 4B.

In addition to the patterning method discussed, the thiol moiety can be anchored on the substrate instead of the vinyl in the surface thiol-ene click reaction. The glass substrate may be initially treated with mercaptopropyl trimethoxysilane (MTS) to produce a thiol-functionalized surface (Figure SI-5), which can then be patterned in a similar manner by using a solution of the desired alkene/alkyne. The free choice between either thiol or vinyl functional groups will provide some flexibility in the choices of functional molecules to immobilize on the substrate. That is, both anchoring molecules, vinyltrimethoxysilane, and mercaptopropyl trimethoxysilane can be paired 
with a wide selection of commercially available counterpart molecules for surface photolithography through thiol-ene coupling reactions.

\section{Preparation and Characterization of Perovskite Thin-Film Microarrays}

A few aprotic organic solvents have been used in perovskite film preparation, ${ }^{49}$ including dimethyl sulfoxide (DMSO), dimethyl formamide (DMF), and $\gamma$-butyrolactone (GBL). To determine the most suitable solvent for this work, the interactions of each solvent with the hydrophilic and hydrophobic regions of the patterned FTO substrate were evaluated through their contact angles, which are summarized in Figure 5. The smaller the contact angle, the better the wettability and the larger affinity. Both DMF and DMSO have very favorable interactions with the ammonium chloride and carboxylic acid functionalized FTO surfaces. GBL interacts less favorably with these hydrophilic surfaces. All three solvents showed high contact angles on the octadecyl-functionalized surface with DMSO having the largest contact angle of $65 \pm 3^{\circ}$. These results indicate that DMSO is the most appropriate solvent for the perovskite patterning project.

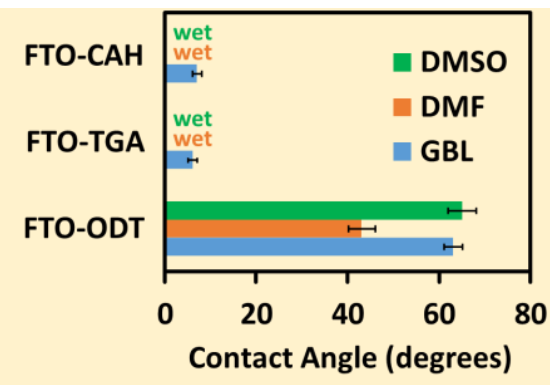

Figure 5. Static contact angles of DMSO, DMF, and GBL on the functionalized FTO substrates. *wet: solvent droplet spread out over the whole substrate with very small contact angles. Error bars are the standard deviation of 9 measurements of 3 independent samples for each substrate type. See supporting information for the data and images.

Perovskite precursor in DMSO solutions can form perovskite microarrays with different quality and reproducibility by spin-coating on patterned glass substrates (Figure SI-7), and 
patterned FTO substrates FTO-TGA-ODT and FTO-CAH-ODT (Figure 6A and 6B). We haven't found a good condition for DMF-based solution yet. The perovskite microarray is observed under a microscope and is further confirmed with fluorescence and x-ray diffraction (XRD) spectroscopy. Methylammonium lead iodide perovskites emit between $700-850 \mathrm{~nm},{ }^{50-58}$ which is consistent with the filter set of our fluorescence microscope. Further confirmation of the perovskite films was done with XRD analysis (Figure SI-6), whose crystal structure is precisely consistent with those reported in the literature. ${ }^{51,54,55,57,59-64}$

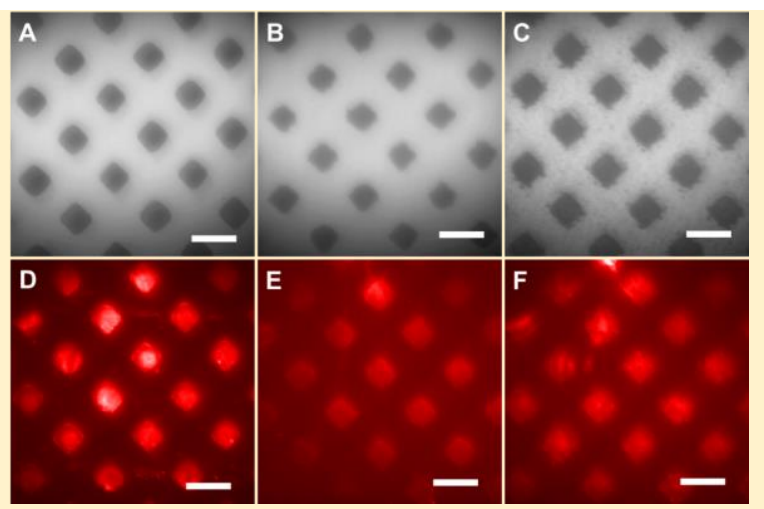

Figure 6. Bright-field microscope images of the perovskite thin film microarrays on patterned (A) FTO-TGA-ODT and (B) FTO-CAH-ODT substrates spin-coated from a DMSO-based precursor; and (C) on an FTO-TGA-ODT spin-coated from a GBL-based precursor. Perovskite thin films are black squares. (D to F) Corresponding fluorescence images of $\mathrm{A}, \mathrm{B}$, and $\mathrm{C}$, respectively. Perovskite is shown red. Scale bars $=40 \mu \mathrm{m}$.

Fluorescence images of the perovskite arrays in both FTO substrates are shown in Figures 6D and 6E excited by a $532 \mathrm{~nm}$ wavelength green laser. The perovskite microarray patterns on the FTO-TGA-ODT substrate with the thin-films closely matching the shape of the square holes in the photomask with nearly $100 \%$ reproducibility of $\sim 10$ repeats. The thin-films from the same solution on the FTO-CAH-ODT surface created less even patterns (Figure 6B) and is less reproducible than on the FTO-TGA-ODT surface. That is, sometimes a patchy perovskite film is deposited on the FTO-CAH-ODT substrate. We don't know the reason yet. A possible explanation for this observation is that the amines on the surface disrupt the formation of the perovskite crystals, 
whereas the - $\mathrm{COOH}$ on the surface of the FTO-TGA-ODT surface can promote the formation of perovskite crystals on its surface by complexing the lead ions. Another possible reason could be trace $\mathrm{CAH}$ has been clicked onto the masked area catalyzed by leaked light or the base. Compared to the dimensions of the square holes in the photomask, all thin-films are slightly smaller $(\sim 28 \mu \mathrm{m}$ comparing to $38 \mu \mathrm{m}, \sim 26 \%$ smaller). This is a sign of liquid contraction during the drying and baking step, which may be improved by optimizing the spin and baking conditions. It is also possibly due to the diffusion of the photo-initiated radicals near the edge of the light spot which may be improved with higher light dose and shorter thiol-ene patterning time.

A GBL-based precursor solvent failed to produce perovskite arrays on an FTO-CAH-ODT surface but works on an FTO-TGA-ODT surface (Figure 6C, 6F). However, isolated small crystals have been observed in the ODT surface under this condition.

Finally, a DMF-based perovskite precursor produces a continuous unpatterned film on both FTO-TGA-ODT and FTO-CAH-ODT surfaces, possibly because the different affinities of DMF on the patterned areas are too small to break the solution into patterned droplets during our spincoating condition.

SEM images (Figure 7) show that the perovskite films deposited from a precursor in DMSO are smooth but slightly wavy, while the films deposited from a precursor in GBL are larger in size, have raised edges and have debris scattered between the films. This suggests that GBL does not work as well as DMSO with the anti-solvent ethyl acetate. DMSO solution should have dried faster than the GBL solution. 

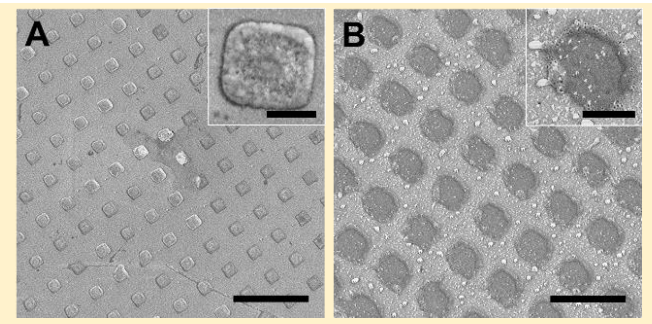

Figure 7. SEM images of perovskite thin-film microarrays on FTO-TGA-ODT deposited from a precursor in DMSO (A) and from a precursor in GBL (B). Spin-coating rate $4000 \mathrm{rpm}$. Perovskites are the squares. Scale bars $150 \mu \mathrm{m}$, inset scale bars $15 \mu \mathrm{m}$.

The perovskite film microarrays were also characterized using AFM (Figure 8). The perovskite films deposited from a precursor in DMSO have a roughness of $37.92 \mathrm{~nm}$ and a maximum film thickness of $\sim 1 \mu \mathrm{m}$ but have a wavy surface and a tapering edge at $4000 \mathrm{rpm}$ spin rate. On the other hand, the perovskite films deposited from a precursor in GBL have a roughness of $37.62 \mathrm{~nm}$, flat and with scattered pinhole defects. The most noticeable feature of this sample is the raised edge of the film, which has a maximum thickness of $\sim 1 \mu \mathrm{m}$ compared to $0.5 \mu \mathrm{m}$ in the middle of the film. This coffee-ring stain effect has been described in literature and occurs due to the higher evaporation rate at the edges compared to the center of the thin liquid film. ${ }^{65,66}$ This results in the net capillary flow of the solution towards the edges which causes a pileup of crystals along the perimeter of the film. ${ }^{67}$ The coffee ring stain effect can be inhibited by controlling the viscosity of the solvent, the temperature of the substrate, ${ }^{68-71}$ and the drying speed. 

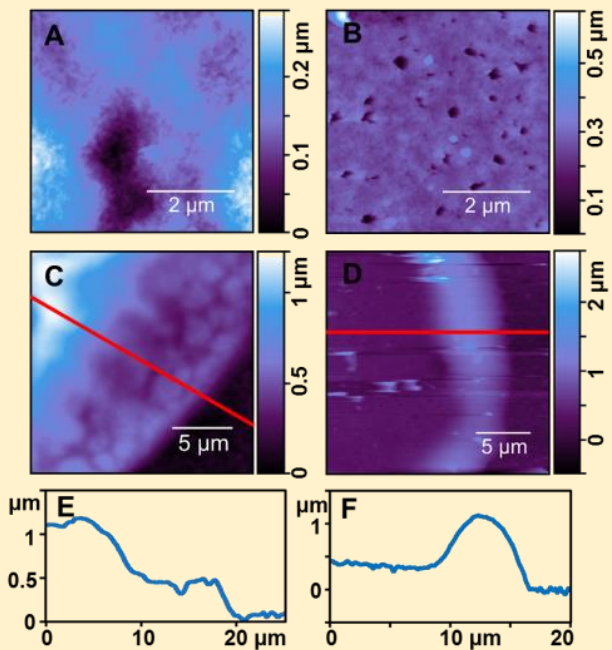

Figure 8. Atomic force microscope images of a perovskite film surface on FTO-TGA-ODT deposited from a precursor solution in DMSO (A) and in GBL (B). Corresponding images of the film edge from a precursor in DMSO (C) and in GBL (D), and (E and F) cross-sectional height traces of the film edges along the red lines in $\mathrm{C}$ and $\mathrm{D}$, respectively. The baseline on the right is the bare FTO surface.

Among many samples and many spin-coatings, some of the deposited perovskite microarrays do not have a good pattern. Fortunately, the perovskite layers can be sonicated and washed off and the patterned substrate can be dried and reused again several times with no degradation observed (Figure 9). This shows that the patterned substrate is robust to the solvent washing, spin-coating, and heat during perovskite baking.

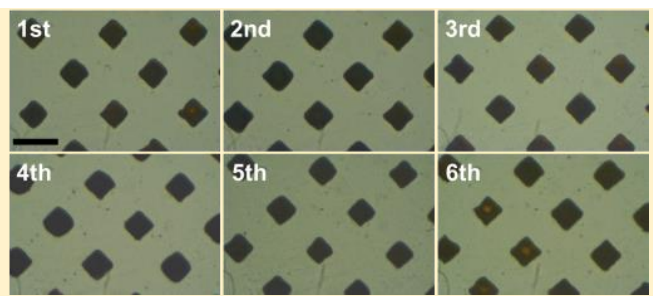

Figure 9. Light microscope images of perovskite thin film microarrays (dark) on an FTO-TGAODT substrate reused six times. The substrate was sonicated in DMSO solvent, rinsed with ethanol and acetone, blown dry with nitrogen, spin-coated with perovskite DMSO solution, and baked during each cycle. Scale bar $50 \mu \mathrm{m}$. 


\section{Conclusions}

Based on the changes in the contact angles after each step of the surface modification and patterning process, and the XPS spectra of the modified substrates, we have shown that we can implement sequential sunlight-initicated thiol-ene click chemistry to create multi-functional patterned oxide substrates. The quality of the patterned substrates was then characterized by labeling the surface-bound amine groups with fluorescent dyes, and by combing DNA strands on a surface patterned with a microarray of positively-charged regions surrounded by negativelycharged areas.

We have also demonstrated the feasibility of a low-cost and versatile method for preparing micropatterned perovskite thin-film microarrays by exploiting the heterogeneous wettability of a chemically patterned FTO substrate. The perovskite precursor solution stays in the area of smaller solvent contact angle forming micro-droplets during the spin-coating and then dried locally after. The FTO-TGA-ODT substrates provide strong anchoring sites for the film, from the favorable interaction of the surface-bound - $\mathrm{COOH}$ groups and the lead ions in the precursor solution. Perovskite precursors in DMSO, GBL, and DMF solution have been spin-coated on FTO glass substrates with TGA-ODT patterns and CAH-ODT patterns. The DMSO solution on TGA-ODT patterned FTO surface shows the best result under our experimental conditions. The patterned substrate can be reused for ideal microarrays. This quick, low-cost, and versatile all-benchtop method for creating perovskite microarrays may be useful for fabricating miniaturized perovskite based optoelectronic devices. 


\section{Acknowledgements}

The authors thank Ohio University startup fund, the National Human Genome Research Institute of the National Institutes of Health Award Number R15HG009972 for funding support, Prof. Michael Jensen, Prof. Katherine Cimatu Group, Prof. Hugh Richardson, and Prof. Andrew Tangonan for instrumental supports and beneficial discussions.

Supporting Information Available. (1) detailed experimental procedures, (2) table and images of solvent contact angles, (3) additional figures of functional surfaces, and (4) XRD spectra of perovskite films.

\section{Associated Content}

\section{Author information}

Corresponding author

Email: chenj@ohio.edu

ORCID: 0000-0001-7381-0918

Notes: The authors declare no competing financial interest.

\section{References}

(1) Quan, L. N.; García de Arquer, F. P.; Sabatini, R. P.; Sargent, E. H. Perovskites for Light Emission. Adv. Mater. 2018, 30 (45), 1801996.

(2) Rajagopal, A.; Yao, K.; Jen, A. K. Y. Toward Perovskite Solar Cell Commercialization: A Perspective and Research Roadmap Based on Interfacial Engineering. Adv. Mater. 2018, 30 (32), 1-45. 
(3) Jagielski, J.; Kumar, S.; Yu, W. Y.; Shih, C. J. Layer-controlled two-dimensional perovskites: Synthesis and optoelectronics. J. Mater. Chem. C 2017, 5 (23), 5610-5627.

(4) Peng, J.; Chen, Y.; Zheng, K.; Pullerits, T.; Liang, Z. Insights into charge carrier dynamics in organo-metal halide perovskites: From neat films to solar cells. Chem. Soc. Rev. 2017, 46 (19), 5714-5729.

(5) Zhao, D.; Yu, Y.; Wang, C.; Liao, W.; Shrestha, N.; Grice, C. R.; Cimaroli, A. J.; Guan, L.; Ellingson, R. J.; Zhu, K.; et al. Low-bandgap mixed tin-lead iodide perovskite absorbers with long carrier lifetimes for all-perovskite tandem solar cells. Nat. Energy 2017, 2 (4), 17018.

(6) Wang, H.; Zhang, X.; Wu, Q.; Cao, F.; Yang, D.; Shang, Y.; Ning, Z.; Zhang, W.; Zheng, W.; Yan, Y.; et al. Trifluoroacetate induced small-grained CsPbBr3 perovskite films result in efficient and stable light-emitting devices. Nat. Commun. 2019, 10 (1), 665.

(7) Luo, J.; Wang, X.; Li, S.; Liu, J.; Guo, Y.; Niu, G.; Yao, L.; Fu, Y.; Gao, L.; Dong, Q.; et al. Efficient and stable emission of warm-white light from lead-free halide double perovskites. Nature 2018, 563 (7732), 541-545.

(8) Gu, Z.; Huang, Z.; Li, C.; Li, M.; Song, Y. A general printing approach for scalable growth of perovskite single-crystal films. Sci. Adv. 2018, 4 (6), 1-9.

(9) Xue, J.; Zhu, Z.; Xu, X.; Gu, Y.; Wang, S.; Xu, L.; Zou, Y.; Song, J.; Zeng, H.; Chen, Q. Narrowband Perovskite Photodetector-Based Image Array for Potential Application in Artificial Vision. Nano Lett. 2018, 18 (12), 7628-7634.

(10) Tong, S.; Gong, C.; Zhang, C.; Liu, G.; Zhang, D.; Zhou, C.; Sun, J.; Xiao, S.; He, J.; Gao, Y.; et al. Fully-printed, flexible cesium-doped triple cation perovskite photodetector. Appl. Mater. Today 2019, 15, 389-397. 
(11) Hörantner, M. T.; Zhang, W.; Saliba, M.; Wojciechowski, K.; Snaith, H. J. Templated microstructural growth of perovskite thin films via colloidal monolayer lithography. Energy Environ. Sci. 2015, 8 (7), 2041-2047.

(12) Jeong, B.; Hwang, I.; Cho, S. H.; Kim, E. H.; Cha, S.; Lee, J.; Kang, H. S.; Cho, S. M.; Choi, H.; Park, C. Solvent-Assisted Gel Printing for Micropatterning Thin OrganicInorganic Hybrid Perovskite Films. ACS Nano 2016, 10 (9), 9026-9035.

(13) Lee, L.; Baek, J.; Park, K. S.; Lee, Y.; Shrestha, N. K.; Sung, M. M. Wafer-scale singlecrystal perovskite patterned thin films based on geometrically-confined lateral crystal growth. Nat. Commun. 2017, 8 (May), 1-8.

(14) Mao, J.; Sha, W. E. I.; Zhang, H.; Ren, X.; Zhuang, J.; Roy, V. A. L.; Wong, K. S.; Choy, W. C. H. Novel Direct Nanopatterning Approach to Fabricate Periodically Nanostructured Perovskite for Optoelectronic Applications. Adv. Funct. Mater. 2017, 27 (10).

(15) Geng, C.; Li, F.; Fan, Y.; Zhang, L.; Shi, S.; Zhang, Z. H.; Zhang, Y.; Xu, S.; Bi, W. Fabrication and Growth Mechanism of Uniform Suspended Perovskite Thin Films. Cryst. Growth Des. 2018, 2-3.

(16) Deng, K.; Liu, Z.; Wang, M.; Li, L. Nanoimprinted Grating-Embedded Perovskite Solar Cells with Improved Light Management. Adv. Funct. Mater. 2019, 29 (19), 1900830.

(17) Kamminga, M. E.; Fang, H.-H.; Loi, M. A.; ten Brink, G. H.; Blake, G. R.; Palstra, T. T. M.; ten Elshof, J. E. Micropatterned 2D Hybrid Perovskite Thin Films with Enhanced Photoluminescence Lifetimes. ACS Appl. Mater. Interfaces 2018, 10 (15), 12878-12885.

(18) Zhang, H.; Liao, Q.; Wu, Y.; Zhang, Z.; Gao, Q.; Liu, P.; Li, M.; Yao, J.; Fu, H. 2D Ruddlesden-Popper Perovskites Microring Laser Array. Adv. Mater. 2018, 30 (15), 1-8.

(19) Waleed, A.; Tavakoli, M. M.; Gu, L.; Hussain, S.; Zhang, D.; Poddar, S.; Wang, Z.; 
Zhang, R.; Fan, Z. All Inorganic Cesium Lead Iodide Perovskite Nanowires with

Stabilized Cubic Phase at Room Temperature and Nanowire Array-Based Photodetectors. Nano Lett. 2017, 17 (8), 4951-4957.

(20) Im, B.; Jun, H.; Lee, K. H.; Lee, J. S. Fabrication of nanoporous MTiO3 (M = Pb, Ba, Sr) perovskite array films with unprecedented high structural regularity. CrystEngComm 2011, 13 (24), 7212-7215.

(21) Baca, A. J.; Roberts, M. J.; Stenger-Smith, J.; Baldwin, L. Manipulating the assembly of perovskites onto soft nanoimprinted titanium dioxide templates. Nanotechnology 2018, 29 (25), 255301.

(22) Zhang, N.; Sun, W.; Rodrigues, S. P.; Wang, K.; Gu, Z.; Wang, S.; Cai, W.; Xiao, S.; Song, Q. Highly Reproducible Organometallic Halide Perovskite Microdevices based on Top-Down Lithography. Adv. Mater. 2017, 29 (15).

Li, R. W.; Kanki, T.; Tohyama, H. A.; Hirooka, M.; Tanaka, H.; Kawai, T. Nanopatterning of perovskite manganite thin films by atomic force microscope lithography. Nanotechnology 2005, 16 (1), 28-31.

(24) Lyashenko, D.; Perez, A.; Zakhidov, A. High-resolution patterning of organohalide lead perovskite pixels for photodetectors using orthogonal photolithography. Phys. Status Solidi Appl. Mater. Sci. 2017, 214 (1), 19-20.

(25) Harwell, J.; Burch, J.; Fikouras, A.; Gather, M. C.; Di Falco, A.; Samuel, I. D. W. Patterning Multicolor Hybrid Perovskite Films via Top-Down Lithography. ACS Nano 2019, 13 (4), 3823-3829.

(26) Lee, W.; Lee, J.; Yun, H.; Kim, J.; Park, J.; Choi, C.; Kim, D. C.; Seo, H.; Lee, H.; Yu, J. W.; et al. High-Resolution Spin-on-Patterning of Perovskite Thin Films for a Multiplexed 
Image Sensor Array. 2017, 1702902, 1-7.

(27) Wu, J.; Chen, J.; Zhang, Y.; Xu, Z.; Zhao, L.; Liu, T.; Luo, D.; Yang, W.; Chen, K.; Hu, Q.; et al. Pinhole-Free Hybrid Perovskite Film with Arbitrarily-Shaped Micro-Patterns for Functional Optoelectronic Devices. Nano Lett. 2017, 17 (6), 3563-3569.

(28) Daus, A.; Roldán-Carmona, C.; Domanski, K.; Knobelspies, S.; Cantarella, G.; Vogt, C.;

Grätzel, M.; Nazeeruddin, M. K.; Tröster, G. Metal-Halide Perovskites for Gate

Dielectrics in Field-Effect Transistors and Photodetectors Enabled by PMMA Lift-Off

Process. Adv. Mater. 2018, 30 (23), 1-8.

(29) Guerra, V. L. P.; Kovaříček, P.; Valeš, V.; Drogowska, K.; Verhagen, T.; Vejpravova, J.; Horák, L.; Listorti, A.; Colella, S.; Kalbáč, M. Selective self-assembly and light emission tuning of layered hybrid perovskites on patterned graphene. Nanoscale 2018, 10 (7), $3198-3211$.

(30) Wu, W.; Wang, X.; Han, X.; Yang, Z.; Gao, G.; Zhang, Y.; Hu, J.; Tan, Y.; Pan, A.; Pan, C. Flexible Photodetector Arrays Based on Patterned CH 3 NH 3 PbI 3- x Cl x Perovskite Film for Real-Time Photosensing and Imaging. Adv. Mater. 2019, 31 (3), 1805913.

(31) Wu, C.; Wang, Z.; Liang, L.; Gui, T.; Zhong, W.; Du, R.; Xie, C.; Wang, L.; Luo, L. Graphene-Assisted Growth of Patterned Perovskite Films for Sensitive Light Detector and Optical Image Sensor Application. Small 2019, 15 (19), 1900730.

(32) Sy Piecco, K. W. E.; Aboelenen, A. M.; Pyle, J. R.; Vicente, J. R.; Gautam, D.; Chen, J. Kinetic Model under Light-Limited Condition for Photoinitiated Thiol-Ene Coupling Reactions. ACS Omega 2018, 3 (10), 14327-14332.

Charles E., H.; Christopher N., B. Thiol-ene click chemistry. Angew. Chem. Int. Ed. (English) 2010, 49 (9), 1540-1573. 
(34) Bai, S.; Da, P.; Li, C.; Wang, Z.; Yuan, Z.; Fu, F.; Kawecki, M.; Liu, X.; Sakai, N.; Wang, J. T.-W.; et al. Planar perovskite solar cells with long-term stability using ionic liquid additives. Nature 2019, 571 (7764), 245-250.

(35) Liu, L.; Mei, A.; Liu, T.; Jiang, P.; Sheng, Y.; Zhang, L.; Han, H. Fully Printable Mesoscopic Perovskite Solar Cells with Organic Silane Self-Assembled Monolayer. J. Am. Chem. Soc. 2015, 137 (5), 1790-1793.

(36) Jung, E. H.; Jeon, N. J.; Park, E. Y.; Moon, C. S.; Shin, T. J.; Yang, T.-Y.; Noh, J. H.; Seo, J. Efficient, stable and scalable perovskite solar cells using poly(3-hexylthiophene). Nature 2019, 567 (7749), 511-515.

Hoyle, C. E.; Lowe, A. B.; Bowman, C. N. Thiol-click chemistry: a multifaceted toolbox for small molecule and polymer synthesis. Chem. Soc. Rev. 2010, 39 (4), 1355-1387.

(38) Hoyle, C. E.; Bowman, C. N. Thiol-Ene Click Chemistry. Angew. Chem. Int. Ed. (English) 2010, 49, 1540-1573.

(39) Bañuls, M. J.; Jiménez-Meneses, P.; Meyer, A.; Vasseur, J. J.; Morvan, F.; Escorihuela, J.; Puchades, R.; Maquieira, Á. Improved Performance of DNA Microarray Multiplex Hybridization Using Probes Anchored at Several Points by Thiol-Ene or Thiol-Yne Coupling Chemistry. Bioconjug. Chem. 2017, 28 (2), 496-506.

(40) Kono, M.; Shiratuchi, R.; Tokashiki, S.; Hayase, S.; Yoshida, Y.; Yamaguchi, Y.; Kubota, $\mathrm{K}$. Increase in photovoltaic performances of dye-sensitized solar cells-Modification of interface between $\mathrm{TiO} 2$ nano-porous layers and F-doped $\mathrm{SnO} 2$ layers. Sol. Energy Mater. Sol. Cells 2008, 92 (6), 646-650.

(41) Bonengel, S.; Bernkop-Schnürch, A. Thiomers - From bench to market. J. Control. Release 2014, 195, 120-129. 
(42) Xie, N.; Ding, X.; Wang, X.; Wang, P.; Zhao, S.; Wang, Z. Journal of Pharmaceutical and Biomedical Analysis Determination of thioglycolic acid in cosmetics by capillary electrophoresis. J. Pharm. Biomed. Anal. 2014, 88, 509-512.

(43) Mohr, P. J.; Taylor, B. N.; Newell, D. B. CODATA recommended values of the fundamental physical constants: 2006. J. Phys. Chem. Ref. Data 2008, 37 (3), 1187-1284.

(44) Zoppe, J. O.; Ataman, N. C.; Mocny, P.; Wang, J.; Moraes, J.; Klok, H. Surface-Initiated Controlled Radical Polymerization: State-of-the-Art, Opportunities, and Challenges in Surface and Interface Engineering with Polymer Brushes. Chem. Rev. 2017, 117 (3), $1105-1318$.

(45) Rittikulsittichai, S.; Park, C. S.; Jamison, A. C.; Rodriguez, D.; Zenasni, O.; Lee, T. R. Bidentate Aromatic Thiols on Gold: New Insight Regarding the Influence of Branching on the Structure, Packing, Wetting, and Stability of Self-Assembled Monolayers on Gold Surfaces. Langmuir 2017, 33 (18), 4396-4406.

(46) NIST X-ray Photoelectron Spectroscopy (XPS) Database.

(47) Chen, Y.; Triola, G.; Waldmann, H. Bioorthogonal Chemistry for Site-Specific Labeling and Surface Immobilization of Proteins. Acc. Chem. Res. 2011, 44 (9), 762-773.

(48) Xi, W.; Scott, T. F.; Kloxin, C. J.; Bowman, C. N. Click Chemistry in Materials Science. Adv. Funct. Mater. 2014, 24 (18), 2572-2590.

(49) Lee, W.; Lee, J.; Yun, H.; Kim, J.; Park, J.; Choi, C.; Kim, D. C.; Seo, H.; Lee, H.; Yu, J. W.; et al. High-Resolution Spin-on-Patterning of Perovskite Thin Films for a Multiplexed Image Sensor Array. Adv. Mater. 2017, 29 (40), 1-7.

(50) Jeon, S.; Zhao, L.; Jung, Y.; Kim, J. W.; Kim, S.; Kang, H.; Jeong, J.; Rand, B. P.; Lee, J. Perovskite Light-Emitting Diodes with Improved Outcoupling Using a High-Index 
Contrast Nanoarray. Small 2019, 15 (8), 1900135.

(51) Li, S.; Li, Y.; Shi, Z.; Lei, L.; Ji, H.; Wu, D.; Xu, T.; Li, X.; Du, G. Fabrication of morphology-controlled and highly-crystallized perovskite microwires for long-term stable photodetectors. Sol. Energy Mater. Sol. Cells 2019, 191 (November 2018), 275-282.

(52) Liu, J.; Li, N.; Jia, J.; Dong, J.; Qiu, Z.; Iqbal, S.; Cao, B. Perovskite films grown with green mixed anti-solvent for highly efficient solar cells with enhanced stability. Sol. Energy 2019, 181 (September 2018), 285-292.

(53) Wang, M.; Fu, Q.; Yan, L.; Guo, P.; Zhou, L.; Wang, G.; Zheng, Z.; Luo, W. Improving the Performance and Reproducibility of Inverted Planar Perovskite Solar Cells Using Tetraethyl Orthosilicate as the Antisolvent. ACS Appl. Mater. Interfaces 2019, 11 (4), 3909-3916.

(54) Wu, C.; Peng, W.; Fang, T.; Wang, B.; Xie, C.; Wang, L.; Yang, W.; Luo, L. Asymmetric Contact-Induced Self-Driven Perovskite-Microwire-Array Photodetectors. Adv. Electron. Mater. 2019, 5 (5), 1900135.

(55) Yi, J.; Zhuang, J.; Ma, Z.; Guo, Z.; Zhou, W.; Zhao, S.; Zhang, H.; Luo, X.; Li, H. Regulated perovskite crystallinity via green mixed antisolvent for e ffi cient perovskite solar cells. Org. Electron. 2019, 69 (November 2018), 69-76.

(56) Zhang, Z.; Luo, X.; Ding, J.; Zhang, J. Preparation of High Quality Perovskite Thin Film in Ambient Air Using Ethylacetate as Anti-solvent. J. Solid State Chem. 2019, 274 (January), 199-206.

(57) Zhang, P.; Yang, F.; Kapil, G.; Ng, C. H.; Ma, T.; Hayase, S. Preparation of Perovskite Films under Liquid Nitrogen Atmosphere for High Efficiency Perovskite Solar Cells. ACS Sustain. Chem. Eng. 2019, 7 (4), 3956-3961. 
Zhizhchenko, A.; Syubaev, S.; Berestennikov, A.; Yulin, A. V; Porfirev, A.; Pushkarev, A.; Shishkin, I.; Golokhvast, K.; Bogdanov, A. A.; Zakhidov, A. A.; et al. Single-Mode Lasing from Imprinted Halide-Perovskite Microdisks. ACS Nano 2019, 13 (4), 41404147.

(59) Hamill, J. C.; Sorli, J. C.; Pelczer, I.; Schwartz, J.; Loo, Y. L. Acid-Catalyzed Reactions Activate DMSO as a Reagent in Perovskite Precursor Inks. Chem. Mater. 2019, 31 (6), $2114-2120$.

(60) Kim, B. G.; Jang, W.; Cho, J. S.; Wang, D. H. Tailoring solubility of methylammonium lead halide with non-stoichiometry molar ratio in perovskite solar cells: Morphological and electrical relationships for high current generation. Sol. Energy Mater. Sol. Cells 2019, 192 (December 2018), 24-35.

(61) Qiu, J.; Mcdowell, L. L.; Shi, Z. Room-Temperature Cubic Perovskite Thin Films by Three-Step All- Vapor Conversion from PbSe to MAPbI 3. Cryst. Growth Des. 2019, 19, 2001-2009.

(62) Rizzo, A.; Lamberti, F.; Buonomo, M.; Wrachien, N.; Torto, L.; Lago, N.; Sansoni, S.; Pilot, R.; Prato, M.; Michieli, N.; et al. Solar Energy Materials and Solar Cells Understanding lead iodide perovskite hysteresis and degradation causes by extensive electrical characterization. Sol. Energy Mater. Sol. Cells 2019, 189 (September 2018), 4352.

(63) Tsai, J.; Cheng, I.; Hsu, C.; Chueh, C.; Chen, J.-Z. Feasibility study of atmosphericpressure dielectric barrier discharge treatment on $\mathrm{CH} 3 \mathrm{NH} 3 \mathrm{PbI} 3$ films for inverted planar perovskite solar cells. Electrochim. Acta 2019, 293, 1-7.

(64) Xiao, Y.; Yang, L.; Han, G.; Li, Y.; Li, M.; Li, H. Effects of methylammonium acetate on 
the perovskite film quality for the perovskite solar cell. Org. Electron. 2019, 65 (November 2018), 201-206.

(65) Shen, X. Y.; Ho, C. M.; Wong, T. S. Minimal Size of Coffee Ring Structure (vol 114B, pg 5273, 2010). J. Phys. Chem. B 2010, 114 (26), 8826.

(66) Weon, B. M.; Je, J. H. Capillary force repels coffee-ring effect. Phys. Rev. E - Stat. Nonlinear, Soft Matter Phys. 2010, 82 (1), 1-4.

(67) Li, Y.; Lan, L.; Xiao, P.; Sun, S.; Lin, Z.; Song, W.; Song, E.; Gao, P.; Wu, W.; Peng, J. Coffee-Ring Defined Short Channels for Inkjet-Printed Metal Oxide Thin-Film Transistors. ACS Appl. Mater. Interfaces 2016, 8 (30), 19643-19648.

(68) Aziz, F.; Ismail, A. F. Spray coating methods for polymer solar cells fabrication: A review. Mater. Sci. Semicond. Process. 2015, 39 (November), 416-425.

(69) Yu, L.; Niazi, M. R.; Ngongang Ndjawa, G. O.; Li, R.; Kirmani, A. R.; Munir, R.; Balawi, A. H.; Laquai, F.; Amassian, A. Programmable and coherent crystallization of semiconductors. Sci. Adv. 2017, 3 (3), e1602462.

(70) Liu, Y.; Li, F.; Qiu, L.; Yang, K.; Li, Q.; Zheng, X.; Hu, H.; Guo, T.; Wu, C.; Kim, T. W. Fluorescent Microarrays of in Situ Crystallized Perovskite Nanocomposites Fabricated for Patterned Applications by Using Inkjet Printing. ACS Nano 2019, 13 (2), 2042-2049.

(71) Yu, X.; Xing, R.; Peng, Z.; Lin, Y.; Du, Z.; Ding, J.; Wang, L.; Han, Y. To inhibit coffee ring effect in inkjet printing of light-emitting polymer films by decreasing capillary force. Chinese Chem. Lett. 2019, 30 (1), 135-138. 